\title{
ON THE NACHBIN COMPACTIFICATION OF PRODUCTS OF TOTALLY ORDERED SPACES
}

\author{
D.C. KENT, DONGMEI LIU
}

\author{
Department of Mathematics \\ Washington State University \\ Pullman, Washington, 99164-3113 \\ and
}

T.A. RICHMOND

Department of Mathematics

Western Kentucky University

Bowling Green, Kentucky 42101

(Received October 18, 1993)

\begin{abstract}
Necessary and sufficient conditions are given for $\beta_{o}(X \times Y)=\beta_{o} X \times \beta_{o} Y$, where $X$ and $Y$ are totally ordered spaces and $\beta_{0} X$ denotes the Nachbin (or Stone-Cech ordered) compactification of $\boldsymbol{X}$.
\end{abstract}

KEY WORDS AND PHRASES. Nachbin compactification, Wallman ordered compactification, totally ordered space, maximal c-filter, strictly first countable space.

1980 AMS SUBJECT CLASSIFICATION CODES: 54 D 35, 54 F 05, 54 B 10.

\section{INTRODUCTION.}

If $X$ is a $T_{3.5}$-ordered space (i.e., an ordered topological space which is "completely regular ordered" in the sense of Nachbin [8]), then $X$ has a largest $T_{2}$-ordered compactification $\beta_{0} X$ which is called the Nachbin (or Stone-Čech ordered) compactification. This compactification, introduced in [8], has been investigated in all of our references except [5].

We are interested in determining when $\beta_{o}(X \times Y)=\beta_{o} X \times \beta_{o} Y$, a problem which has not previously received attention in the literature. The methods used by Glicksberg [5] to solve the corresponding problem for the Stone-Cech compactification do not appear to be fruitful when applied to the Nachbin compactification. Therefore, at this preliminary stage of our investigation, we have focused our attention on the case where $X$ and $Y$ are "totally ordered spaces", where a totally ordered space is defined to be a totally ordered set with a convex, $T_{2}$-ordered topology (not necessarily the order topology). Our main result, Theorem 5.6, gives a simple condition for totally ordered spaces $X$ and $Y$ which is necessary and sufficient for $\beta_{o}(X \times Y)=\beta_{0} X \times \beta_{o} Y$. 
Our solution to the aforementioned problem makes extensive use of the Wallman ordered compactification $w_{0} X$, introduced in [2]. In Section 3 we show that for any $T_{3.5}$-ordered space $X$, $\beta_{0} X$ can be obtained from $w_{0} X$ via a certain quotient construction, and this result is employed in the proof of our main theorem. We also make use of the fact that $w_{0} X=\beta_{0} X$ for any totally ordered space $X$.

We first prove a preliminary version of our product theorem in Section 4 under the assumption that the totally ordered spaces $X$ and $Y$ are "strictly first countable". (A totally ordered space is defined to be strictly first countable if every neighborhood filter and every maximal closed-convex filter has a countable filter base.) Surprisingly, the condition which "works" in the strictly first countable case also "works" in the general case (see Theorems 4.4 and 5.6). If $X$ and $Y$ are strictly first countable, then $\beta_{o}(X \times Y)=\beta_{o} X \times \beta_{o} Y$ and $w_{o}(X \times Y)=w_{o} X \times w_{o} Y$ are equivalent statements. We do not know if this equivalence holds for arbitrary totally ordered spaces $X$ and $Y$.

\section{PRELIMINARIES.}

Let $(X, \leq)$ be a poset. For a non-empty subset $A$ of $X$, we define $d(A)=\{y \in X: y \leq$ $x$ for some $x \in A\}$ to be the decreasing hull of $A$; the increasing hull $i(A)$ is defined dually. We shall write $d(x)$ and $i(x)$ in place of $d(\{x\})$ and $i(\{x\})$. A set $A$ is increasing (respectively, decreasing) if $A=i(A)$ (respectively, $A=d(A)$ ); a set which is either increasing or decreasing is said to be monotone. For any $A \subseteq X, A^{\wedge}=i(A) \cap d(A)$ is called the convex hull of $A$, and $A$ is convex if $A=A^{\wedge}$.

Let $\boldsymbol{F}(X)$ denote the set of all filters on a set $X$. We always use the term filter to mean a proper set filter. If $\mathcal{F}$ and $\mathcal{G}$ are filters on $X$ such that $F \cap G \neq \emptyset$, for all $F \in \mathcal{F}$ and $G \in \mathcal{G}$, then $\mathcal{F} \vee \mathcal{G}$ denotes the filter generated by $\{F \cap G: F \in \mathcal{F}, G \in \mathcal{G}\}$; if, on the other hand, $\mathcal{F}$ and $\mathcal{G}$ contain disjoint sets, we say that $\mathcal{F} \vee \mathcal{G}$ fails to exist. A filter $\mathcal{F}$ is free if there is no point common to all the sets in $\mathcal{F}$. A filter which is not free is said to be fixed; in particular, the symbol $\dot{x}$ will denote the fixed ultrafilter generated by $x \in X$. For any filter $\mathcal{F}$ on $X$, the filter $\mathcal{F}^{\wedge}$ generated by sets of the form $\left\{F^{\wedge}: F \in \mathcal{F}\right\}$ is called the convex hull of $\mathcal{F}$.

An ordered topological space, or simply an ordered space, is a triple $(X, \leq, \tau)$, where $(X, \leq)$ is a poset and $\tau$ a convex topology on $(X, \leq)$ (i.e., $\tau$ is a topology which has a subbase consisting of monotone open sets). Note that every ordered space is locally convex in the sense that every neighborhood filter has a base of convex open sets. When there is no danger of confusion, we refer to the ordered space $(X, \leq, \tau)$ simply as $X$. If $X$ and $Y$ are ordered spaces, a map $f: X \rightarrow Y$ is increasing (respectively, decreasing) if $x \leq y$ in $X$ implies $f(x) \leq f(y)$ (respectively, $f(y) \leq f(x)$ ) in $Y$. A continuous, increasing map is called an ordered topological morphism, or more briefly a morphism. A bijective morphism whose inverse is also a morphism is called an ordered topological isomorphism, or more briefly an isomorphism. Let $C I^{*}(X)$ (respectively, $C D^{*}(X)$ ) denote the set of all morphisms (respectively, continuous, decreasing maps) from an ordered space $X$ into $[0,1]$.

An ordered space $X$ is $T_{1}$-ordered if $i(x)$ and $d(x)$ are closed sets, for all $x \in X ; X$ is $T_{2}$-ordered if the partial order relation " $\leq$ " is closed in $X \times X$. An ordered space $X$ is $T_{3.5}$-ordered (completely regular ordered in [8]) if the following conditions are satisfied: (1) If $x \in X, A$ is a closed subset of $X$, and $x \notin A$, then there is $f \in C I^{*}(X)$ and $g \in C D^{*}(X)$ such that $f(x)=g(x)=0$ and $f(y) \vee g(y)=1$, for all $y \in A$; (2) If $x \leq y$ in $X$, there is $f \in C I^{*}(X)$ such that $f(y)=0$ and $f(x)=1$. The $T_{3.5}$-ordered spaces are precisely those which allow $T_{2}$-ordered compactifications (see [3] and [8]). An ordered space $X$ is normally ordered (see [8]) if, whenever $A$ and $B$ are disjoint closed sets, with $A$ increasing and $B$ decreasing, there are disjoint open sets $U$ and $V$, 
with $U$ increasing and $V$ decreasing, such that $A \subseteq U$ and $B \subseteq V$. An ordered space which is both normally ordered and $T_{1}$-ordered is said to be $T_{4}$-ordered.

Given a $T_{3.5}$-ordered space $X$, there is a largest $T_{2}$-ordered compactification of $X$ called the Nachbin (or Stone-Cech ordered) compactification, denoted by $\beta_{o} X$. The standard construction of $\beta_{0} X$ involves embedding $X$ in the "ordered cube" $[0,1]^{C I^{\bullet}(X)}$ where the latter space has the usual product order and topology. This compactification is characterized by the following well-known extension theorem (see $[3]$ or $[8]$ ).

THEOREM 2.1. If $X$ is a $T_{3.5}$-ordered space, $Y$ is a compact, $T_{2}$-ordered space, and $f: X \rightarrow Y$ is a morphism, then there is a unique morphism $f^{\prime}: \beta_{o} X \rightarrow Y$ such that the diagram

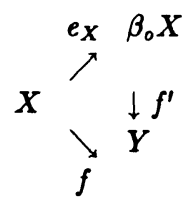

commutes, where $e_{X}: X \rightarrow \beta_{o} X$ is the canonical embedding.

We next review the construction of the Wallman ordered compactification. Let $X$ be an ordered space and $A^{-} X$; let $D(A)$ (respectively, $I(A)$ ) be the smallest closed, decreasing (respectively, closed, increasing) set that contains $A$. Let $A^{\Delta}=I(A) \cap D(A)$; if $A=A^{\Delta}$ then $A$ is called a $c$-set. One may verify that the collection of all $c$-sets on $X$ is closed under arbitrary intersections. It is obvious that $c$-sets are closed and convex, but not all closed, convex subsets of $X$ are c-sets. If $\mathcal{F} \in \boldsymbol{F}(X)$, let $D(\mathcal{F})$ (respectively, $I(\mathcal{F})$ ) denote the filter on $X$ generated by $\{D(F): F \in \mathcal{F}\}$ (respectively, $\{I(F): F \in \mathcal{F}\}$ ). The filter $\mathcal{F}^{\Delta}=I(\mathcal{F}) \vee D(\mathcal{F})$ is generated by sets of the form $F^{\Delta}$, for $F \in \mathcal{F}$; if $\mathcal{F}=\mathcal{F}^{\Delta}$, then $\mathcal{F}$ is called a $c$-filter. One may easily verify (using Zorn's Lemma) that every $c$-filter is coarser than a maximal $c$-filter.

Let $X$ be a $T_{1}$-ordered space, and let $w_{0} X$ be the set of all maximal $c$-filters on $X$. A partial order "§» for $w_{0} X$ is defined as follows: $\mathcal{G} \Longleftrightarrow I(\mathcal{F}) \subseteq \mathcal{G}$ and $D(\mathcal{G}) \subseteq \mathcal{F}$. We also assign to $w_{0} X$ the topology with closed subbase $C^{*}=\left\{A^{*}: A=A^{\Delta}\right\}$, where $A^{*}=\left\{\mathcal{F} \in w_{0} X: A \in \mathcal{F}\right\}$. Then $w_{0} X$, with the order and topology just described, is an ordered space which is compact and $T_{1}$ (but generally not $T_{1}$-ordered). If $\varphi_{X}: X \rightarrow w_{0} X$ is defined by $\varphi_{X}(x)=\dot{x}$, for all $x \in X$, then $\varphi_{X}$ is an isomorphic embedding, and consequently $\left(w_{0} X, \varphi_{X}\right)$ is an ordered compactification of $X$. Furthermore, we have the following extension property (see [2] or [6]).

THEOREM 2.2. Let $X$ be a $T_{1}$-ordered space, $Y$ a compact, $T_{2}$-ordered space, and $f: X \rightarrow Y$ a morphism. Then there is a unique morphism $\bar{f}: w_{0} X \rightarrow Y$ such that the diagram

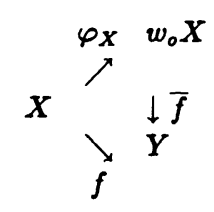

commutes.

An ordered space $X$ is defined to be a $c$-space if $i(A)$ and $d(A)$ are closed subsets of $X$ whenever $A \subseteq X$ is a $c$-set. The next theorem is proved in [6].

THEOREM 2.3. For a $T_{3.5}$-ordered space $X$, the following statements are equivalent. 
(1) $\quad w_{0} X$ is $T_{2}$-ordered

(2) $w_{0} X=\beta_{0} X$

(3) $\quad X$ is a $T_{4}$-ordered $c$-space.

3. $\beta_{0} X$ AS A QUOTIENT OF $w_{0} X$.

Throughout this section, $X$ will denote an arbitrary $T_{3.5}$-ordered space and $\left(w_{0} X, \varphi\right)$ the Wallman ordered compactification of $X$.

If $f \in C I^{\bullet}(X)$, then there exists, by Theorem 2.2, a unique morphism $f^{\wedge}: w_{0} X \rightarrow[0,1]$ extending $f$. We define an equivalence relation $R$ on $w_{0} X$ as follows: $R=\left\{(\mathcal{F}, \mathcal{g}) \in w_{0} X \times\right.$ $w_{0} X: f^{\wedge}(\mathcal{F})=f^{\wedge}(\mathcal{G})$, for all $\left.f \in C I^{\bullet}(X)\right\}$. The set $\left\{[\mathcal{F}]: \mathcal{F} \in w_{0} X\right\}$ of $R$-equivalence classes is denoted by $w_{0} X / R$; let $\sigma: w_{0} X \rightarrow w_{0} X / R$ be the canonical projection map. We endow $w_{0} X / R$ with the quotient topology derived from $w_{0} X$ and $\sigma$, and with the partial order $[\mathcal{F}] \leq_{R}[\mathcal{G}] \Longleftrightarrow f^{\wedge}(\mathcal{F}) \leq f^{\wedge}(\mathcal{G})$ in $[0,1]$, for all $f \in C I^{*}(X)$. The set $w_{0} X / R$, with this order and topology, is an ordered space which, for convenience, we shall call $\mu_{o} X$. One easily verifies that $\sigma: w_{o} X \rightarrow \mu_{o} X$ is a morphism.

LEMMA 3.1. $\mu_{o} X$ is a compact, $T_{2}$-ordered space.

PROOF. Obviously, $\mu_{0} X$ is compact. We recall (see [8]) that an ordered space is $T_{2}$-ordered $\Longleftrightarrow$, whenever $x \not y$, there are disjoint neighborhoods $U$ and $V$ of $x$ and $y$, respectively, such that $U$ is increasing and $V$ is decreasing.

For each $f \in C I^{\bullet}(X)$, define $f^{\prime}: \mu_{0} X \rightarrow[0,1]$ by $f^{\prime}([\mathcal{F}])=f^{\wedge}(\mathcal{F})$, for all $[\mathcal{F}] \in \mu_{o} X$; it is easy to verify that $f^{\prime}$ is a well defined morphism, and therefore $\left\{f^{\prime}: f \in C I^{*}(X)\right\} \subseteq C I^{*}\left(\mu_{o} X\right)$. If $[\mathcal{F}] \mathbb{Z}_{R}[\mathcal{G}]$, there is $f \in C I^{\bullet}(X)$ such that $f^{\wedge}(\mathcal{F}) \leq f^{\wedge}(\mathcal{G})$ in $[0,1]$, and hence $f^{\prime}([\mathcal{F}])>$ $f^{\prime}([\mathcal{G}])$ in $[0,1]$. Let $f^{\prime}([\mathcal{F}])-f^{\prime}([\mathcal{G}])=\varepsilon>0$, and let $U=\left(f^{\prime}\right)^{-1}\left(\left(f^{\prime}([\mathcal{F}])-\varepsilon / 3,1\right]\right)$ and $V=\left(f^{\prime}\right)^{-1}\left(\left[0, f^{\prime}([\mathcal{G}])+\varepsilon / 3\right)\right)$. Then $U$ and $V$ are disjoint open nieghborhoods separating $[\mathcal{F}]$ and $[\mathcal{G}]$ such that $U$ is increasing and $V$ decreasing. Therefore $\mu_{0} X$ is $T_{2}$-ordered.

By Theorem 1.2, there is a unique morphism $\bar{e}: w_{0} X \rightarrow \beta_{0} X$ such that the diagram

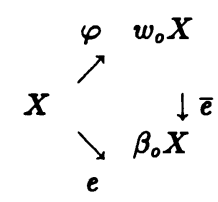

commutes. Also, for any $f \in C I^{\bullet}(X)$, there is a unique $\bar{f} \in C I^{\bullet}\left(\beta_{0} X\right)$ such that $f=\bar{f} \circ e$. Note that $f^{\wedge}=\bar{f} \circ \bar{e}$, since these maps agree on the dense subspace $\varphi(X)$ of $w_{0} X$, and hence on $w_{0} X$.

LEMMA 3.2. For $\mathcal{F}, \mathcal{G} \in w_{0} X,(\mathcal{F}, \mathcal{G}) \in R \Longleftrightarrow \bar{e}(\mathcal{F})=\bar{e}(\mathcal{G})$.

PROOF. If $(\mathcal{F}, \mathcal{G}) \in R$, then $f^{\wedge}(\mathcal{F})=f^{\wedge}(\mathcal{G})$, for all $f \in C I^{*}(X)$, and hence $\bar{f}(\bar{e}(\mathcal{F}))=$ $\bar{f}(\bar{e}(\mathcal{G}))$ for all $f \in C I^{\bullet}(X)$. This implies $\bar{e}(\mathcal{F})=\bar{e}(\mathcal{G})$, since $\beta_{0} X$ is $T_{3.5}$-ordered and hence $C I^{*}\left(\beta_{o} X\right)=\left\{\bar{f}: f \in C I^{*}(X)\right\}$ separates points in $\beta_{o} X$. Conversely, if $\bar{e}(\mathcal{F})=\bar{e}(\mathcal{G})$, then $f^{\wedge}(\mathcal{F})=\bar{f}(\bar{e}(\mathcal{F}))=\bar{f}(\bar{e}(\mathcal{G}))=f^{\wedge}(\mathcal{G})$ for all $f \in C I^{\bullet}(X)$, which implies $(\mathcal{F}, \mathcal{G}) \in R$.

THEOREM 3.3. For any $T_{3.5}$-ordered space $X, \beta_{0} X$ and $\mu_{0} X$ are isomorphic. 
PROOF. Let $e^{*}: \mu_{o} X \rightarrow \beta_{o} X$ be defined by $e^{*}([\mp])=\bar{e}(\mp)$, for all $₹ \in w_{o} X$.

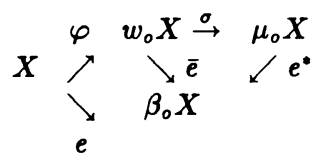

It follows from Lemma 3.2 that $e^{\bullet}$ is a well-defined bijection. Since $\mu_{0} X$ has the quotient topology induced by $\sigma$ and $\bar{e}=e^{\bullet} \circ \sigma$ is continuous, $e^{\bullet}$ is continuous. Since $\mu_{o} X$ is compact and $\beta_{o} X$ is $T_{2}, e^{\bullet}$ is a homeomorphism.

To check that $e^{\bullet}$ is an order-isomorphism, let $[\mathcal{F}] \leq_{R}[\mathcal{G}]$ in $\mu_{o} X$. Then, for all $f \in C I^{\bullet}(X), f^{\wedge}(\mathcal{F})$ $\leq f^{\wedge}(\mathcal{G})$ in $[0,1]$, and hence $\bar{f}(\bar{e}(\mathcal{F})) \leq \bar{f}(\bar{e}(\mathcal{G}))$, for all $f \in C I^{\bullet}(X)$. Thus $\bar{e}(\mathcal{F}) \leq \bar{e}(\mathcal{G})$ in $\beta_{0} X$, and consequently $e^{\bullet}(\sigma(\mathcal{F}))=e^{\bullet}([\mathcal{F}]) \leq e^{\bullet}([\mathcal{G}])=e^{\bullet}(\sigma(\mathcal{G}))$. This argument is reversible, and consequently both $e^{*}$ and $\left(e^{*}\right)^{-1}$ are increasing maps.

\section{A PRELIMINARY PRODUCT THEOREM.}

Compactifications of totally ordered spaces are studied in [1] and [7], and we begin this section by summarizing some relevent results from [7]. We define a totally ordered space $X$ to be a $T_{2}$ ordered space whose partial order is a total order (i.e., if $x, y \in X$, then $x \leq y$ or $y \leq x$ ). It is easy to show that a totally ordered space is a $T_{4}$-ordered $c$-space in which the $c$-sets are precisely the closed, convex sets. Consequently, by Theorem 2.3, the compactifications $w_{0} X$ and $\beta_{o} X$ of a totally ordered space $X$ exist and are equal. Furthermore, every $T_{2}$-ordered compactification of a totally ordered space is itself a totally ordered space.

For a totally ordered space $X$, we use the equivalence of $\beta_{o} X$ and $w_{o} X$ to describe the compactification points of $\beta_{o} X$ as maximal $c$-filters. It is shown in [7] that, in a totally ordered space $X$, the maximal $c$-filters are precisely the convex hulls of ultrafilters; the non-convergent maximal $c$-filters on $X$ are called singularities. Given a singularity $\mathcal{F}$, let $\mathcal{F}^{\dagger}=\cup\left\{F^{\dagger}: F \in \mathcal{F}\right\}$, where $F^{\dagger}$ denotes the set of upper bounds of $F$, and $F^{\downarrow}=\cup\left\{F^{\downarrow}: F \in \mathcal{F}\right\}$, where $F^{\downarrow}$ is the set of lower bounds of $F$. The convex sets $\mathcal{F}^{\dagger}$ and $F^{\downarrow}$ partition $X$, and so exactly one of these sets is in $₹$. If $\mathcal{F}^{\dagger} \in \mathcal{F}$ (respectively, $\mathcal{F}^{\downarrow} \in \mathcal{F}$ ) we say that $\mathcal{F}$ is a decreasing (respectively, increasing) singularity.

A totally ordered space $\boldsymbol{X}$ is strictly first countable if every neighborhood filter and every singularity has a countable filter base. If $\mathcal{F}$ is an increasing singularity with a countable filter base, then there is a strictly increasing sequence $x_{1}<x_{2}<x_{3}<\cdots$ in $X$ such that $\mathcal{F}$ is the convex hull of the filter of sections of $\left(x_{n}\right)$; similarly, each decreasing singularity with a countable filter base is likewise derived from a strictly decreasing sequence in $X$.

If $X$ and $Y$ are totally ordered spaces, then $X \times Y$ (with the product order and product topology) is a $T_{3.5}$-ordered space, but not generally a $c$-space. For instance, it is shown in [4] that if $X$ is the real line with the usual order and topology and $Y$ is any totally ordered space whose underlying poset is the real line, then $X \times Y$ is a $c$-space $\Longleftrightarrow$ the topology for $Y$ is the usual topology. Thus, in general, $w_{o}(X \times Y)$ fails to be $T_{2}$-ordered and hence $w_{o}(X \times Y)$ and $\beta_{o}(X \times Y)$ are non-equivalent compactifications (see Theorem 2.3). The next two lemmas are due to Margaret A. Gamon.

LEMMA 4.1. If $X$ and $Y$ are totally ordered spaces, $A$ a $c$-set in $X$, and $B$ a $c$-set in $Y$, then $A \times B$ is a $c$-set in $X \times Y$.

PROOF. It is clear that $i(A \times B)=i(A) \times i(B)$. Since totally ordered spaces are $c$ spaces, $i(A)$ and $d(A)$ are both closed, and so $i(A)=I(A)$ and $d(A)=D(A)$. Therefore, 
$i(A \times B)=I(A) \times I(B)$ is a closed, increasing set containing $A \times B$, and hence $i(A \times B)=$ $I(A \times B)=I(A) \times I(B)$. Similarly, $D(A \times B)=D(A) \times D(B)$, and therefore $I(A \times B) \cap D(A \times B)=$ $(I(A) \times I(B)) \cap(D(A) \times D(B))=(I(A) \cap D(A)) \times(I(B) \cap D(B))=A \times B$. Thus $A \times B$ is a $c$-set in $X \times Y$.

LEMMA 4.2. Let $X$ and $Y$ be totally ordered spaces, and let $F$ be a singularity on $X$ and $G$ a singularity on $Y$ such that either both are increasing singularities or both are decreasing singularities. Then $₹ \times \mathcal{G}$ is a maximal $c$-filter on $X \times Y$.

PROOF. Assume that $\mathcal{F}$ and $\mathcal{G}$ are both increasing singularities on $X$ and $Y$, respectively. By Lemma 4.1, $₹ \times \mathcal{G}$ is a $c$-filter on $X \times Y$. Let $X^{\prime}=\left\{x \in X: \dot{x} \lesssim \mathcal{F}\right.$ in $\left.w_{0} X\right\}$ and $Y^{\prime}=$ $\left\{y \in Y: \dot{y} \lesssim \mathcal{G}\right.$ in $\left.w_{0} Y\right\}$ be totally ordered subspaces of $X$ and $Y$, respectively. Let $\mathcal{F}^{\prime}$ and $\mathcal{G}^{\prime}$ be the restrictions of $\mathcal{F}$ and $\mathcal{G}$ to $X^{\prime}$ and $Y^{\prime}$, respectively. It is easy to verify that $\mathcal{F}^{\prime}$ and $\mathcal{G}^{\prime}$ are increasing singularities on $X^{\prime}$ and $Y^{\prime}$, respectively, and that $\left(f^{\prime}\right)^{\dagger}=\left(\mathcal{G}^{\prime}\right)^{\dagger}=\emptyset$. It is also easy to verify that $₹ \times \mathcal{G}$ is a maximal $c$-filter on $X \times Y \Longleftrightarrow \mathcal{F}^{\prime} \times \mathcal{G}^{\prime}$ is a maximal $c$-filter on $X^{\prime} \times Y^{\prime}$. Therefore we shall assume, without loss of generality, that $\mathcal{F}$ and $\mathcal{G}$ are increasing singularities on $X$ and $Y$, respectively, such that $\mathcal{F}^{\dagger}=\mathcal{G}^{\dagger}=\emptyset$.

Let $\forall$ be a $c$-filter on $X \times Y$ such that $₹ \times g \subseteq H$. Let $H \in \forall$ be a convex set, and let $(a, b) \in H \cap(F \times G)$, where $F \in \mathcal{F}$ and $G \in \mathcal{G}$. For any $c \in X$ and $d \in Y$ such that $a \leq c$ and $b \leq d$, we must have $(c, d) \in H$, since otherwise $i(c) \times i(d)$ would be a member of $\mathcal{F} \times \mathcal{G}$ disjoint from $H$. This implies that $i(a) \times i(b) \subseteq H$. But $i(a) \times i(b) \in \mathcal{F} \times \mathcal{G}$, and so $\forall \subseteq \mathcal{F} \times \mathcal{G}$. It follows that $\mathcal{H}=\mathcal{F} \times \mathcal{G}$, and consequently $\mathcal{F} \times \mathcal{G}$ is a maximal $c$-filter on $X \times Y$.

In general, the conclusion of Lemma 4.2 is not valid if one of the singularities is increasing and the other decreasing. Indeed, the next lemma establishes that if $\mathcal{F}$ is an increasing singularity on $X$ and $\mathcal{G}$ a decreasing singularity on $Y$, both with countable filter bases, then $\mathcal{F} \times \mathcal{G}$ is not a maximal $c$-filter on $X \times Y$.

LEMMA 4.3. Let $X$ and $Y$ be totally ordered spaces. Let $\left(x_{n}\right)$ be a strictly increasing sequence in $X$ and $\left(y_{n}\right)$ a strictly decreasing sequence in $Y$. Let $S=\left\{\left(x_{2 n-1}, y_{2 n-1}\right): n \in N\right\}$ and $T=\left\{\left(x_{2 n}, y_{2 n}\right): n \in N\right\}$. Then there is $g \in C I^{*}(X \times Y)$ such that $g(i(S))=1$ and $g(d(T))=0$.

PROOF. Choose $x_{0} \in X$ such that $x_{0}<x_{1}$. (In case $x_{1}$ is the least element of $X$, replace the original sequence $\left(x_{n}\right)$ by $\left(x_{n}^{\prime}\right)$, where $x_{n}^{\prime}=x_{n+1}$.) Since $X$ and $Y$ are both $T_{4}$-ordered spaces, we can apply Theorem 1, page 30, [8] to obtain, for each $n \in N$, a function $f_{2 n-1} \in C I^{*}(X)$ such that $f_{2 n-1}(a)=0$ if $a \leq x_{2 n-2}$ and $f_{2 n-1}(b)=1$ if $b \geq x_{2 n-1}$, and another function $f_{2 n} \in C I^{*}(Y)$ such that $f_{2 n}(c)=0$ if $c \leq y_{2 n}$ and $f_{2 n}(d)=1$ if $d \geq y_{2 n-1}$.

We next define a family of functions in $C I^{*}(X \times Y)$ as follows:

$$
\begin{aligned}
H_{2}(x, y) & =\left(f_{1}(x) f_{2}(y)\right) \wedge 1 \\
H_{4}(x, y) & =\left[H_{2}(x, y)+f_{3}(x) f_{4}(y)\right] \wedge 1 \\
& * \\
& * \\
& * \\
H_{2 n}(x, y) & =\left[H_{2 n-2}(x, y)+f_{2 n-1}(x) f_{2 n}(y)\right] \wedge 1
\end{aligned}
$$

For $k \in N$, we define $S^{2 k-1}=\left\{\left(x_{2 n-1}, y_{2 n-1}\right): n \leq k\right\}$ and $T^{2 k}=\left\{\left(x_{2 n}, y_{2 n}\right): n \leq k\right\}$. It is easy to verify that $H_{2 k}\left(d\left(T^{2 k}\right)\right)=0$ and $H_{2 k}\left(i\left(S^{2 k-1}\right)\right)=1$. 
Finally, let

$$
g(x, y)=\lim _{n \rightarrow \infty}\left[\left(H_{2 n}(x, y)+f_{2 n+1}(x) f_{2 n+2}(y)\right) \wedge 1\right] .
$$

It is clear that $g$ is an increasing map from $X \times Y$ into $[0,1]$ such that $g(x, y)=1$ if $(x, y) \in$ $i(S)$ and $g(x, y)=0$ if $(x, y) \in d(T)$. By considering the possible cases, one may also verify that for every $(x, y) \in X \times Y, g(x, y)$ is the infimum of the constant function 1 and a finite sum of continuous functions. Thus $g \in C I^{*}(X \times Y)$, as desired.

In the proof of the next theorem we will need some additional notation. Let $X$ and $Y$ be totally ordered spaces, and consider the following diagram:

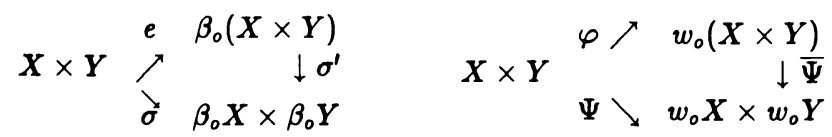

where, in the notation of Section $2, e=e_{X \times Y}, \sigma=e_{X} \times e_{Y}, \varphi=\varphi_{X \times Y}$, and $\Psi=\varphi_{X} \times \varphi_{Y}$ are the canonical embedding maps. Since $w_{0} X=\beta_{0} X$ and $w_{0} Y=\beta_{0} Y, \beta_{0} X \times \beta_{0} Y=w_{0} X \times w_{0} Y$ is a compact, $T_{2}$-ordered space, and $\sigma^{\prime}$ and $\bar{\Psi}$ are the unique, continuous, increasing extension maps whose existence is guaranteed in Theorems 2.1 and 2.2.

Observe that $\beta_{o}(X \times Y)=\beta_{o} X \times \beta_{o} Y$ (respectively, $\left.w_{o}(X \times Y)=w_{o} X \times w_{o} Y\right) \Longleftrightarrow \sigma^{\prime}$ (respectively, $\bar{\Psi}$ ) is an injective map.

THEOREM 4.4. If $X$ and $Y$ are strictly first countable, totally ordered spaces, then the following statements are equivalent.

(1) $\quad w_{0} X \times w_{o} Y=w_{0}(X \times Y)$.

(2) $\beta_{0} X \times \beta_{0} Y=\beta_{o}(X \times Y)$.

(3) If either $X$ or $Y$ has an increasing (or decreasing) singularity, then the other space contains no strictly decreasing (or strictly increasing) sequence.

PROOF. (1) $\Rightarrow$ (2). For totally ordered spaces $X$ and $Y, \beta_{o} X=w_{0} X$ and $\beta_{o} Y=w_{0} Y$. Thus $w_{o}(X \times Y)=\beta_{o} X \times \beta_{0} Y$, and the latter space is $T_{2}$-ordered. By Theorem 2.3, $w_{o}(X \times Y)=$ $\beta_{o}(X \times Y)=\beta_{o} X \times \beta_{o} Y$.

(2) $\Rightarrow(3)$. Assume $X$ has an increasing singularity $\mathcal{G}$ and that $Y$ contains a strictly decreasing sequence $\left(y_{n}\right)$. Note that $\mathcal{G}$ is a compactification point in $\beta_{0} X=w_{0} X$. If $\left(y_{n}\right)$ converges to $y_{0}$ in $Y$, then we define $\gamma=\left(\mathcal{G}, \varphi_{Y}\left(y_{o}\right)\right)$; if $\left(y_{n}\right)$ fails to converge in $Y$, let $\not$ be the decreasing singularity in $Y$ which is the convex hull of the filter of sections of $\left(y_{n}\right)$, and let $\gamma=(\mathcal{G}, \mathcal{H})$. In either case, $\gamma$ is a compactification point of $\beta_{o} X \times \beta_{o} Y$.

Next, let $\left(x_{n}\right)$ be a strictly increasing sequence in $X$ obtained by choosing a denumerable, nested filter base $\left\{G_{n}: n \in N\right\}$ for $\mathcal{G}$ and choosing $x_{n} \in G_{n}$, for all $n \in N$, such that $x_{1}<x_{2}<$ $x_{3}<\cdots$. Then clearly $\mathcal{G}$ is the convex hull of the filter of sections of the sequence $\left(x_{n}\right)$.

Consider the filter $\mathcal{F}$ of sections of the sequence $\left(x_{n}, y_{n}\right)$ on $X \times Y$; let $\mathcal{F}_{1}$ be the filter generated by the subsequence $\left(x_{2 n-1}, y_{2 n-1}\right)$ and $f_{2}$ the filter generated by the subsequence $\left(x_{2 n}, y_{2 n}\right)$. If $S=\left\{\left(x_{2 n-1}, y_{2 n-1}\right): n \in N\right\}$ and $T=\left\{\left(x_{2 n}, y_{2 n}\right): n \in N\right\}$, then $S \in \mathcal{F}_{1}$ and $T \in \mathcal{F}_{2}$. Regardless of whether or not $\left(y_{n}\right)$ converges in $Y, \sigma(\mathcal{F})$ (and hence also $\sigma\left(F^{\Delta}\right)$ ) converges to $\gamma$ in $\beta_{0} X \times \beta_{0} Y$.

Let $\mathcal{M}_{1}$ and $\mathcal{M}_{2}$ be maximal $c$-filters on $X \times Y$ such that $f_{1}^{\Delta} \subseteq \mathcal{M}_{1}$ and $f_{2}^{\Delta} \subseteq \mathcal{M}_{2}$. By Lemma 4.3, there is $g \in C I^{*}(X \times Y)$ such that $g(i(S))=1$ and $g(d(T))=0$. Since $g^{-1}(\{1\}) \in \mathcal{M}_{1}$ and $g^{-1}(\{0\}) \in M_{2}$, it follows that $g\left(M_{1}\right)$ converges to 1 and $g\left(\mathcal{M}_{2}\right)$ converges to 0 in $[0,1]$. Using Theorem 3.3, we identify $\beta_{0}(X \times Y)$ with the quotient space $\mu_{0}(X \times Y)$; thus we regard the 
$R$-equivalence classes $\left[\mathcal{M}_{1}\right]$ and $\left[\mathcal{M}_{2}\right]$ (defined in the second paragraph of Section 3 ) as elements of $\beta_{0}(X \times Y)$. Since $g^{\wedge}\left(\mathcal{M}_{1}\right) \neq g^{\wedge}\left(\mathcal{M}_{2}\right)$ (where $g^{\wedge} \in C I^{*}\left(w_{0}(X \times Y)\right.$ ) is the unique extension of $\left.g \in C I^{*}(X \times Y)\right), \quad\left[\mathcal{M}_{1}\right]$ and $\left[\mathcal{M}_{2}\right]$ are distinct equivalence classes (i.e., distinct elements in $\beta_{o}(X \times Y)$ ). But $\sigma\left(\mathcal{M}_{1}\right)$ and $\sigma\left(\mathcal{M}_{2}\right)$ are both finer than $\sigma\left(\mathcal{F}^{\Delta}\right)$, so both of these filters converge to $\gamma$ in $\beta_{0}(X \times Y)$. Thus $\sigma^{\prime}\left(\left[\mathcal{M}_{1}\right]\right)=\sigma^{\prime}\left(\left[\mathcal{M}_{2}\right]\right)$, and consequently $\sigma^{\prime}$ is not injective.

(3) $\Rightarrow(1)$. If neither $X$ nor $Y$ has a singularity, then $X$ and $Y$ are both compact, and so $X \times Y=w_{0} X \times w_{0} Y=w_{0}(X \times Y)$.

Assume that $w_{0} X \times w_{0} Y$ contains a compactification point $\gamma=(\mathcal{F}, \mathcal{G})$. We will show that $\bar{\Psi}^{-1}(\gamma)$ is a singleton in $w_{0}(X \times Y)$, implying that $\bar{\Psi}$ is injective. There are three possible cases to consider. If $\mathcal{F}$ is a singularity in $X$ and $\mathcal{G}$ a singularity in $Y$, then Condition (3) and the assumption of strict first countability for $X$ and $Y$ imply that $\mathcal{F}$ and $\mathcal{G}$ are either both increasing singularities or both decreasing singularities. By Lemma 4.2, $\mp \times \mathcal{G}$ is a maximal $c$-filter on $X \times Y$. Thus $\bar{\Psi}^{-1}(\gamma)=\{\mathcal{F} \times \mathcal{G}\}$ is a single compactification point in $w_{0}(X \times Y)$. If $\mathcal{F}$ is a singularity and $\mathcal{G}=\varphi_{Y}(y)$ for some $y \in Y$, then one easily verifies that $\mathcal{F} \times \dot{y}$ is a non-convergent maximal $c$-filter on $X \times Y$, and $\bar{\Psi}^{-1}(\gamma)=\{\xi \times \dot{y}\}$ is again a singleton compactification point in $w_{o}(X \times Y)$. The same reasoning applies if $\mathcal{G}$ is a singularity in $Y$ and $\mathcal{F}=\varphi_{X}(x)$ for some $x \in X$.

We conclude that the quotient map $\bar{\Psi}: w_{o}(X \times Y) \rightarrow w_{o} X \times w_{0} Y$ is injective, and therefore $w_{o}(X \times Y)=w_{o} X \times w_{o} Y$.

If $X$ is the real line with any convex, $T_{2}$-ordered topology and $Y$ is any strictly first countable, totally ordered space, it follows from the preceding theorem that $\beta_{o}(X \times Y)=\beta_{o} X \times \beta_{o} Y$ iff $Y$ is finite. If $N$ is the set of natural numbers with the usual order and the discrete topology, $\beta_{o}(N \times N)=\beta_{o} N \times \beta_{o} N$ follows by Theorem 3.4; note that $N \times N$ is not pseudo-compact and thus $\beta(N \times N) \neq \beta N \times \beta N$. If, on the other hand, $N^{*}$ is the set of negative integers with their usual order and the discrete topology, $\beta_{o}\left(N \times N^{*}\right) \neq \beta_{0} N \times \beta_{o} N^{*}$. Indeed, it is easy to see that $\beta_{o} N \times \beta_{o} N^{*}$ has cardinality $\aleph_{o}$, whereas it can be shown that $\beta_{o}\left(N \times N^{*}\right)$ has cardinality $2^{2^{\mathrm{K}_{0}}}$.

\section{THE PRODUCT THEOREM.}

Throughout this section, the symbols $X$ and $Y$ will represent arbitrary totally ordered spaces. Our main theorem (Theorem 5.6) establishes that Condition (3) of Theorem 4.4 (stated in slightly different terms) is necessary and sufficient for $\beta_{o}(X \times Y)=\beta_{o} X \times \beta_{o} Y$ in the general case. The proof of Theorem 5.6 is based on five rather technical lemmas, for which we need some additional notation and terminology.

Let $\mathcal{F}$ be an increasing singularity on $X$ and $\xi$ an ordinal number. Recall that $\mathcal{F}$ is also a compactification point in $w_{o} X=\beta_{o} X$. We say that $\mathcal{F}$ has order $\xi$ if there is a strictly increasing net $\left(x_{\wedge}\right)_{\wedge<\xi}$ on $X$ such that the net $\left(\dot{x}_{\wedge}\right)_{\wedge<\xi}$ in $\beta_{0} X$ converges to $\mathcal{F}$, and $\xi$ is the least such ordinal. The order of a decreasing singularity on $X$ is defined dually. If $\xi$ is the order of any singularity on $X$, then clearly $\xi$ is an infinite ordinal and the least ordinal of its cardinality. In a strictly first countable, totally ordered space, every singularity has order $\omega$, the least infinite ordinal.

Next, let $y \in Y$. If no strictly increasing net in $Y$ converges to $y$, we say that $y$ has left order 0 . If for some ordinal $\xi$, there is a strictly increasing net $\left(y_{\wedge}\right)_{\wedge<\xi}$ converging to $y$ in $Y$ and $\xi$ is the least such ordinal, we say that $y$ has left order $\xi$. The right order for $y$ is defined dually.

If $z \in Y$ and $y<z$, let $[y, z]=\{a \in Y: y \leq a \leq z\}, \quad[y, z)=\{a \in Y: y \leq a<z\}$, and $(y, z]=\{a \in Y: y<a \leq z\}$. If $y \in Y$ has left order $\rho>0$ and $\left(y_{\wedge}\right)_{\wedge<\rho}$ is a strictly increasing 
net converging to $y$, we denote by $\nu_{\ell}(y)$ the filter on $Y$ generated by $\left\{\left[y_{\wedge}, y\right]: \wedge<\rho\right\} ; V_{\ell}(y)$ is called the left neighborhood filter at $y$, and we set $\nu_{\ell}(y)=\dot{y}$ in case $\rho=0$. Likewise, if $y$ has right order $\xi>0$ and $\left(z_{\wedge}\right)_{\wedge<\xi}$ is a strictly decreasing net converging to $y$, the right neighborhood filter $\nu_{r}(y)$ is generated by $\left\{\left[y, z_{\wedge}\right]: \wedge<\xi\right\}$; again we set $\nu_{r}(y)=\dot{y}$ if $\xi=0$. Furthermore, if $\xi>0$ we denote by $\mathcal{V}_{r}^{\prime}(y)$ the filter on $Y$ generated by $\left\{\left(y, z_{\wedge}\right]: \wedge<\xi\right\}$. Note that $\mathcal{V}_{\ell}(y) \cap \mathcal{V}_{r}(y)$ is the usual neighborhood filter at $y$.

We shall also need additional interval notation pertaining to singularities. If $\mathcal{F}$ is an increasing singularity on $X$ and $x \in X$ is such that $\dot{x} \lesssim \mathcal{F}$ in $w_{0} X$, we define $[x, \mathcal{F}\rangle=\{a \in X: x \leq a$ in $X$ and $\dot{a} \lesssim \mathcal{F}$ in $\left.w_{0} X\right\}$ and $(x, \mathcal{F}\rangle=\{x, \mathcal{F}\rangle \backslash\{x\}$. In case $\mathcal{G}$ is a decreasing singularity on $Y$ and $y \in Y$ is such that $\mathcal{G} \lesssim \dot{y}$ in $w_{0} Y$, let $\langle\mathcal{G}, y]=\left\{a \in Y: a \leq y\right.$ in $Y$ and $\mathcal{G} \lesssim \dot{a}$ in $\left.w_{o} Y\right\}$ and let $\langle\mathcal{G}, y)=\langle\mathcal{G}, y] \backslash\{y\}$. If $\mathcal{F}$ has order $\xi$ and $\left(x_{\wedge}\right)_{\wedge<\xi}$ is a strictly increasing net such that $\left(\dot{x}_{\wedge}\right)_{\wedge<\xi}$ converges to $\mathcal{F}$ in $w_{0} X$, then each of the sets $\left\{\left|x_{\wedge}, \mathcal{F}\right\rangle: \wedge\langle\xi\}\right.$ and $\left\{\left(x_{\wedge}, \mathcal{F}\right\rangle: \wedge\langle\xi\}\right.$ is a filter base for $\mathcal{F}$. Likewise, if $\mathcal{G}$ has order $\eta$ and $\left(y_{\wedge}\right)_{\wedge<\eta}$ is a strictly decreasing net in $Y$ such that $\left(\dot{y}_{\wedge}\right)_{\wedge<\eta}$ converges to $\mathcal{G}$ in $w_{0} Y$, then each of the sets $\left\{\left\langle\mathcal{G}, y_{\wedge}\right]: \wedge<\eta\right\}$ and $\left\{\left\langle\mathcal{G}, y_{\wedge}\right): \wedge<\eta\right\}$ are filter bases for $\mathcal{G}$.

LEMMA 5.1. Let $\mathcal{F}$ be an increasing singularity on $X$ of order $\xi>\omega$, let $\mathcal{G}$ be a decreasing singularity on $Y$ of order $\eta>\omega$, and assume that every strictly increasing sequence on $X$ and every strictly decreasing sequence on $Y$ is convergent. If $\mathcal{L}$ and $\mathcal{M}$ are maximal $c$-filters on $X \times Y$, both finer than $₹ \times \mathcal{G}$, then for all $f \in C I^{*}(X \times Y), f(\mathcal{L})$ and $f(\mathcal{M})$ converge to the same limit in $[0,1]$.

PROOF. Suppose there is $f \in C I^{*}(X \times Y)$ such that $f(\mathcal{L})$ converges to $a, f(\mathcal{M})$ converges to $b$, and $a \neq b$ in $[0,1]$. Let $U$ and $V$ be disjoint neighborhoods of $a$ and $b$, respectively, and choose closed sets $L \in \mathcal{L}$ and $M \in \mathcal{M}$ such that $f(L) \subseteq U$ and $f(M) \subseteq V$. Then, choose the following points in $X \times Y$ :

$$
\begin{aligned}
& \left(a_{o}, b_{o}\right) \in L \cap\left(\left(x_{o}, \mathcal{F}\right\rangle \times\left\langle\mathcal{G}, y_{o}\right)\right) \\
& \left(c_{o}, d_{o}\right) \in M \cap\left(\left(a_{o}, \mathcal{F}\right\rangle \times\left\langle\mathcal{G}, b_{o}\right)\right) \\
& \left(a_{1}, b_{1}\right) \in L \cap\left(\left(c_{o}, \mathcal{F}\right\rangle \times\left\langle\mathcal{G}, d_{o}\right)\right) \\
& \left(c_{1}, d_{1}\right) \in M \cap\left(\left(a_{1}, \mathcal{F}\right\rangle \times\left\langle\mathcal{G}, b_{1}\right)\right) \\
& \vdots \\
& \vdots
\end{aligned}
$$

Continuing in this way we obtain sequences $\left(a_{n}, b_{n}\right)$ in $L$ and $\left(c_{n}, d_{n}\right)$ in $M$ such that

$$
\begin{aligned}
& a_{o}<c_{o}<a_{1}<c_{1}<\cdots<a_{n}<c_{n}<\cdots \\
& b_{0}>d_{0}>b_{1}>d_{1}>\cdots>b_{n}>d_{n}>\cdots
\end{aligned}
$$

Under the assumptions of the lemma, the sequence $a_{o}, c_{o}, a_{1}, c_{1}, \cdots$ converges to some $x_{0}$ in $X$, and the sequence $b_{o}, d_{o}, b_{1}, d_{1}, \cdots$ converges to some $y_{o}$ in $Y$. Thus $\left(x_{o}, y_{o}\right) \in L \cap M$, contrary to the fact that $f(L) \cap f(M)=\phi$.

LEMMA 5.2. Let $\mathcal{F}$ be an increasing singularity of order $\xi>\omega$ on $X$, and let $y \in Y$ have right order $\eta>\omega$. Assume that every strictly increasing sequence on $X$ and every strictly decreasing sequence on $Y$ is convergent. If $\mathcal{L}$ and $\mathcal{M}$ are maximal $c$-filters on $X \times Y$, both finer than $\mathcal{F} \times \mathcal{V}_{r}^{\prime}(y)$, then for all $f \in C I^{*}(X \times Y), f(\mathcal{L})$ and $f(\mathcal{M})$ converge to the same limit in $[0,1]$.

PROOF. The proof of Lemma 5.2 is essentially identical to that of Lemma 5.1. 
LEMMA 5.3. Let $\mathcal{F}$ be an increasing singularity on $X$ of order $\xi \geq \omega$ and let $y \in Y$ have left order $\eta \geq 0$. If $\mathcal{M}$ is a maximal $c$-filter on $X \times Y$ finer than $\mathcal{F} \times \mathcal{V}_{\ell}(y)$, then $\mathcal{M}=\mathcal{F} \times \dot{y}$.

PROOF. Let $\left(y_{\mu}\right)_{\mu<\eta}$ be a strictly increasing net on $Y$ converging to $y$; thus $\nu_{\ell}(y)$ has a filter base $\left.\left\{\mid y_{\mu}, y\right]: \mu<\eta\right\}$. Note that $\mathcal{F}$ has a filter base of the form $\left\{\left|x_{\wedge}, \mathcal{F}\right\rangle: \wedge<\xi\right\}$, where $\left(x_{\wedge}\right)_{\wedge<\xi}$ is a strictly increasing net in $X$.

Choose a $c$-set $M \in M$ such that $\left[x_{o}, \mp\right) \times\left[y_{o}, y\right] \supseteq M$. For each $\wedge<\xi$ and $\mu<\eta$, there is $\left(a_{\wedge \mu}, b_{\wedge \mu}\right) \in M \cap\left(\left[x_{\wedge}, \mathcal{F}\right\rangle \times\left[y_{\mu}, y\right]\right)$. Let $(a, b) \in M$ be arbitrary; we shall show that $(a, y) \in M$. If $b=y$ there is nothing more to show, so assume the contrary, and choose ordinals $\rho<\eta$ and $\tau<\xi$ such that $b<y_{\rho}$ and $a<x_{\tau}$. Then $(a, b) \leq\left(a, b_{\tau \rho}\right) \leq\left(a_{\tau \rho}, b_{\tau \rho}\right)$, and so by convexity of $M$, $\left(a, b_{\tau \rho}\right) \in M$. Since $y_{\rho} \leq b_{\tau \rho}, \quad\left(a, y_{\rho}\right) \in M$. Indeed this reasoning implies that $\left(a, y_{\mu}\right) \in M$ for all $\mu<\eta$ such that $\rho \leq \mu$. Since $M$ is closed, $(a, y) \in M$. Using again the convexity of $M$, we deduce that $\{a, \mathcal{F}\rangle \times\{y\} \subseteq M$ and that $[a, \mathcal{F}\rangle \in \mathcal{F}$. Thus $\mathcal{F} \times \dot{y} \geq \mathcal{M}$, and since both are maximal $c$-filters, equality holds.

LEMMA 5.4. Let $\mathcal{F}$ be an increasing singularity on $X$ of order $\xi \geq \omega$, and let $y \in Y$ have right order $\eta \geq \omega$. If $\xi \neq \eta$ and $\mathcal{M}$ is a maximal $c$-filter on $X \times Y$ finer than $\mathcal{F} \times \mathcal{V}_{r}(y)$, then $\mathcal{M}=\mp \times \dot{y}$.

PROOF. Let $\left(x_{\lambda}\right)_{\lambda<\xi}$ be a strictly increasing net in $X$ such that $\left\{\left|x_{\lambda}, \mathcal{F}\right\rangle: \lambda<\xi\right\}$ is a filter base for $\mathcal{F}$. Let $\left(y_{\mu}\right)_{\mu<\eta}$ be a strictly decreasing net in $Y$ converging to $y$ such that $\left\{\left[y, y_{\mu}\right]: \mu<\eta\right\}$ is a filter base for $\nu_{r}(y)$. Let $M \in M$ be a closed, convex set such that $M \subseteq\left[x_{0}, \mathcal{F}\right\rangle \times\left[y, y_{0}\right]$.

CASE 1. $\eta<\xi$. If $0 \leq \lambda<\eta$, choose $\left(a_{\lambda}, b_{\lambda}\right) \in M \cap\left(\left[x_{\lambda}, \mathcal{F}\right\rangle \times\left[y, y_{\lambda}\right]\right)$ such that $\left(a_{\lambda}\right)_{\lambda<\eta}$ is strictly increasing in $X$ and $\left(b_{\lambda}\right)_{\lambda<\eta}$ is strictly decreasing in $Y$. Next, choose ordinal $\rho$ such that $\eta<\rho<\xi$, and choose $\left(a_{\rho}, b_{\rho}\right) \in M \cap\left[x_{\rho}, \mathcal{F}\right\rangle \times\left[y, y_{o}\right]$ such that $a_{\lambda}<a_{\rho}$, for all $\lambda<\eta$. Let $\Lambda=\left\{\lambda<\eta: b_{\lambda}<b_{\rho}\right\}$. Using the convexity of $M,\left(a_{\lambda}, b_{\lambda}\right) \in M$, for all $\lambda \in \Lambda$ and $\left(a_{\rho}, b_{\rho}\right) \in M$ implies that $\left(a_{\rho}, b_{\lambda}\right) \in M$, for all $\lambda \in \Lambda$. Since $\left(b_{\lambda}\right)_{\lambda \in \Lambda}$ converges to $y$ in $Y$ and $M$ is closed, $\left(a_{\rho}, y\right) \in M$. This reasoning leads to the conclusion that $\left[a_{\rho}, \mathcal{F}\right\rangle \times\{y\} \subseteq M$, and hence $\mathcal{F} \times \dot{y}=\mathcal{M}$.

CASE 2. $\xi<\eta$. For each $\lambda<\eta$, choose $\left(a_{\lambda}, b_{\lambda}\right) \in M \cap\left[x_{o}, \mathcal{F}\right\rangle \times\left[y, y_{\lambda}\right]$ such that $\left(b_{\lambda}\right)_{\lambda<\eta}$ is strictly decreasing; thus $\left(b_{\lambda}\right)_{\lambda<\eta}$ converges to $y$ in $Y$. For each $\lambda<\eta$, let $\mu_{\lambda}$ be the least ordinal such that $a_{\lambda}<x_{\mu \lambda}$. Note that $\left\{\mu_{\lambda}: \lambda<\eta\right\} \subseteq\{\rho: \rho<\xi\}$. Considering the net $\left(x_{\mu_{\lambda}}\right)_{\lambda<\eta}$, we observe that since each $\mu_{\lambda}<\xi$, there is some $\rho \in\left\{\mu_{\lambda}: \lambda<\mu\right\}$ such that the term $x_{\rho}$ occurs $|\eta|$ times in the net $\left(x_{\mu_{\lambda}}\right)_{\lambda<\eta}$, where $|\eta|$ is the cardinality of $\eta$.

Now choose a point $(a, b) \in M$ such that $a>x_{\rho}$. Then $a>a_{\lambda}$, for all $\lambda<\mu$ such that $\rho=\mu_{\lambda}$. If $\Lambda=\left\{\lambda<\eta: \rho=\mu_{\lambda}\right\}$, then $|\Lambda|=|\eta|$ and $\left(b_{\lambda}\right)_{\lambda \in \Lambda}$ converges to $y$ in $Y$. We shall show that $(a, y) \in M$. Assuming $b \neq y$, let $\Lambda^{\prime}=\left\{\lambda \in \Lambda: b_{\lambda}<b\right\}$; then $\left|\Lambda^{\prime}\right|=\mid \eta$ ! and $\left(b_{\lambda}\right)_{\lambda \in \Lambda^{\prime}}$ converges to $y$ in $Y$. Using the now familiar argument based on $M$ being closed and convex, we deduce that $(a, y) \in M$. This argument can again be extended to show $[a, \mathcal{F}\rangle \times\{y\} \subseteq M$, where $[a, \mathcal{F}\rangle \in \mathcal{F}$, and consequently $\mathcal{M}=\mathcal{F} \times \dot{y}$.

LEMMA 5.5. Let $\mathcal{F}$ be an increasing singularity on $X$ of order $\xi>\omega$, and let $y \in Y$ have right order $\xi$. If $\mathcal{M}$ is a maximal $c$-filter on $X \times Y$ finer than $\mathcal{F} \times \mathcal{V}_{r}(y)$, then for all $f \in C I^{*}(X \times Y), f(\mathcal{M})$ and $f(\mathcal{F} \times \dot{y})$ converge to the same limit in $[0,1]$.

PROOF. Assume $\mathcal{F}$ has filter base $\left\{\left|x_{\lambda}, \mathcal{F}\right\rangle: \lambda<\xi\right\}$ as in the preceding proof. Suppose there 
is $f \in C I^{*}(X \times Y)$ and $\mathcal{M} \geq \mathcal{F} \times \mathcal{V}_{r}(y)$ such that $f(\mathcal{F} \times \dot{y})$ converges to $a \in[0,1]$ and $f(\mathcal{M})$ converges to some point in $[0,1]$ other than a. Since $\mathcal{M} \neq \mathcal{F} \times \dot{y}$, it follows that $\mathcal{M} \geq \mathcal{F} \times \mathcal{V}_{r}^{\prime}(y)$. We shall obtain a contradiction by constructing a maximal $c$-filter $\mathcal{L} \geq \mathcal{F} \times \mathcal{V}_{r}^{\prime}(y)$ such that, for each $L \in \mathcal{L}, f(L)$ intersects every neighbhorhood of $a$ in $[0,1]$. It follows that $f(\mathcal{L})$ converges to $a$ in $[0,1]$ and hence, by Lemma 5.2, $f(\mathcal{M})$ converges to $a$, a contradiction.

Let $\left\{U_{n}: n \in N\right\}$ be a nested neighborhood base for $a \in[0,1]$, where each $U_{n}$ is a closed interval. Since $f(\mathcal{F} \times \dot{y})$ converges to $a$ and $\xi>\omega$, we can find $\rho<\xi$ such that $\left[x_{\rho}, \mathcal{F}\right\rangle \in \mathcal{F}$ and $\left[x_{\rho}, F\right\rangle \times\{y\} \subseteq C$, where $C=\cap\left\{U_{n}: n \in N\right\}$ is a $c$-set in $X \times Y$. Let $\Lambda=\{\lambda: \rho \leq \lambda<\xi\}$. Let $\left(y_{\lambda}\right)_{\lambda<\xi}$ be a strictly decreasing net converging to $y$ in $Y$. For each $\lambda \in \Lambda$, there is $\mu_{\lambda}<\xi$ such that $\left(x_{\lambda}, z\right) \in C$, for all $z \in\left[y, y_{\mu_{\lambda}}\right]$. Choose a strictly decreasing net $\left(y_{\eta_{\lambda}}\right)_{\lambda \in \Lambda}$ such that $y_{\eta_{\lambda}} \in\left[y, y_{\mu_{\lambda}}\right)$ for all $\lambda \in \Lambda$; then $\left(y_{\eta_{\lambda}}\right)_{\lambda \in \Lambda}$ converges to $y$ and $\left(x_{\lambda}, y_{\eta_{\lambda}}\right)_{\lambda \in \Lambda}$ is a net in $C$. Let $\mathcal{K}$ be the filter of sections of the net $\left(x_{\lambda}, y_{\eta_{\lambda}}\right)_{\lambda \in \Lambda}$, and let $\mathcal{L}$ be any maximal $c$-filter finer than $\mathcal{K}^{\Delta}=I(\mathcal{K}) \cap D(K)$. Since $C$ is a $c$-set, $C \in K^{\Delta}$ and hence $C \in \mathcal{L}$. Thus $f(\mathcal{L})$ converges to $a$ in $[0,1]$. Since each set of the form $\left[x_{\mu}, \exists\right\rangle \times\left[y, y_{\mu}\right]$, for $\mu<\xi$, contains an element of the net $\left(x_{\lambda}, y_{\eta_{\lambda}}\right)_{\lambda \in \Lambda}, \mathcal{K}$ is finer than the $c$-filter $\mathcal{F} \times \mathcal{V}_{r}(y)$, and consequently $\mathcal{L} \geq K^{\Delta} \geq \mathcal{F} \times \mathcal{V}_{r}(y)$. To complete the proof, it remains to show that $\mathcal{L} \neq \mathcal{F} \times \dot{y}$, and therefore that $\mathcal{L} \geq \mathcal{F} \times \mathcal{V}_{r}^{\prime}(y)$.

If $\mathcal{L}=\mathcal{F} \times \dot{y}$, then each $S \in K^{\Delta}$ contains a set of the form $F \times\{y\}$ for some $F \in \mathcal{F}$. Let $S=K^{\Delta}$, where $K \in \mathcal{K}$ has the form $K=\left\{\left(x_{\lambda}, y_{\eta_{\lambda}}\right): \lambda \geq \mu\right\}$ for some $\mu \in \Lambda$. If a set of the form $F \times\{y\} \subseteq K^{\Delta}$ for some $F \in \mathcal{F}$, then there is an ordinal $\delta \geq \mu$ such that $\left[x_{\delta}, \mathcal{F}\right\rangle \subseteq F$; thus $\left\{x_{\delta}, \mathcal{F}\right\rangle \times\{y\} \subseteq K^{\Delta}$. Let $\epsilon$ be any ordinal such than $\delta<\epsilon<\xi$. Then $D=\left\{(x, z): x \geq x_{\epsilon}\right\} \cup\left\{(x, z): z \geq y_{\eta_{e}}\right\}$ is a $c$-set in $X \times Y$ containing $K$, and therefore $K^{\Delta} \subseteq D$. But $\left\{x_{\delta}, x_{\epsilon}\right) \times\{y\} \cap D=\emptyset$, and so $\left[x_{\delta}, \mathcal{F}\right\rangle \times\{y\} \nsubseteq K^{\Delta}$. The assumption that $\mathcal{L}=\mathcal{F} \times \dot{y}$ is hereby contradicted, and the proof of the lemma is complete.

THEOREM 5.6. Let $X$ and $Y$ be totally ordered spaces. Then $\beta_{0}(X \times Y)=\beta_{0} X \times \beta_{o} Y \Longleftrightarrow$ the following condition (*) is satisfied: $\left({ }^{*}\right)$ If either $X$ or $Y$ contains an increasing (or decreasing) singularity of order $\omega$, then the other space contains no strictly decreasing (or strictly increasing) sequence.

PROOF. If $\beta_{o}(X \times Y)=\beta_{o} X \times \beta_{o} Y$, the proof that (2) $\Rightarrow(3)$ in Theorem 3.4 establishes condition $\left({ }^{*}\right)$.

Conversely, assume $\left({ }^{*}\right)$ and consider the diagram

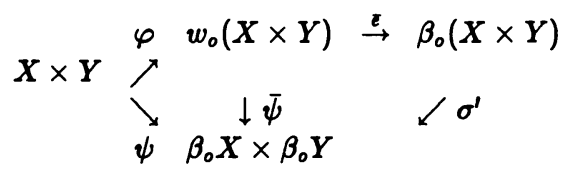

To show $\beta_{o}(X \times Y)=\beta_{0} X \times \beta_{o} Y$, it is sufficient to show that $\left(\sigma^{\prime}\right)^{-1}(\alpha)$ is a singleton for each compactification point $\alpha$ in $\beta_{0} X \times \beta_{0} Y$. Two cases must be considered.

CASE 1. $\alpha=(\mathcal{F}, \mathcal{G})$, where $\mathcal{F}$ and $\mathcal{G}$ are singularities on $X$ and $Y$, respectively. If $\mathcal{F}$ and $\mathcal{G}$ are either both increasing or both decreasing, it follows by Lemma 4.2 that $\left(\sigma^{\prime}\right)^{-1}(\alpha)$ is a singleton. So, without loss of generality, assume that $\mathcal{F}$ is an increasing singularity of order $\xi$ and $\mathcal{G}$ is a decreasing singularity of order $\eta$. If $\xi=\omega$, then the existence of a decreasing singularity on $Y$ implies the existence of a strictly decreasing sequence in $Y$, contrary to condition $\left({ }^{*}\right)$. Thus there is no loss of generality in assuming $\xi \geq \eta>\omega$; we also assume, in view of $\left({ }^{*}\right)$, that every strictly increasing sequence on $X$ converges in $X$ and every strictly decreasing sequence on $Y$ converges in $Y$.

The preceding observations allow us to conclude, using Lemma 5.1, that if $\mathcal{L}$ and $\mathcal{M}$ are in 
$\bar{\psi}^{-1}(\alpha)$, then for all $f \in C I^{*}(X \times Y), f(\mathcal{L})$ and $f(\mathcal{M})$ converge to the same limit in $[0,1]$. Thus, by Theorem 3.3, $\bar{e}(\mathcal{L})=\bar{e}(\mathcal{M})$ in $\beta_{o}(X \times Y)$; in other words, $\left(\sigma^{\prime}\right)^{-1}(\alpha)$ is a singleton.

CASE 2. $\alpha=(\mathcal{F}, \mathcal{G})$, where one member of this pair is a singularity and the other a fixed ultrafilter. Without loss of generality, we assume that $\mathcal{F}$ is an increasing singularity of order $\xi \geq \omega$, and $\mathcal{G}=\dot{y}$, where $y \in Y$ has left order $\rho \geq 0$ and right order $\eta \geq 0$.

We first observe that $\mathcal{F} \times \dot{y}$ is a maximal $c$-filter on $X \times Y$ and obviously $\mathcal{F} \times \dot{y} \in \bar{\psi}^{-1}(\alpha)$. To complete the proof, it is sufficient to show that if $\mathcal{M}$ is any maximal $c$-filter on $X \times Y$ finer than $\mathcal{F} \times\left(\mathcal{V}_{r}(y) \cap \mathcal{V}_{\ell}(y)\right)$, then $f(\mathcal{M})$ and $f(\mathcal{F} \times \dot{y})$ converge to the same limit in $[0,1]$ for all $f \in C I^{*}(X \times Y)$; then the desired conclusion that $\left(\sigma^{\prime}\right)^{-1}(\alpha)$ is a singleton follows, as in Case 1, from Theorem 3.3. Furthermore, since $\mathcal{V}_{r}(y)$ and $\mathcal{V}_{\ell}(y)$ are both $c$-filters on $Y, \mathcal{M} \geq \mathcal{F} \times\left(\mathcal{V}_{r}(y) \cap \mathcal{V}_{\ell}(y)\right)$ implies either $\mathcal{M} \geq \mp \times \mathcal{V}_{r}(y)$ or $\mathcal{M} \geq \mp \times \mathcal{V}_{\ell}(y)$.

If $\mathcal{M}$ is a maximal $c$-filter finer than $\mathcal{F} \times V_{\ell}(y)$, it follows by Lemma 5.3 that $\mathcal{M}=\mathcal{F} \times \dot{y}$, and the conclusion is trivial. We thus assume, for the remainder of the proof, that $\mathcal{M} \geq \mathcal{F} \times \mathcal{V}_{r}(y)$. If $\eta=0$, then again $\mathcal{M}=\mathcal{F} \times \dot{y}$ and the proof is complete. If $\eta \geq \omega$, we observe that $Y$ contains a strictly decreasing sequence; thus by condition $\left({ }^{*}\right), \xi>\omega$, and furthermore every strictly increasing sequence on $X$ must converge. The existence of the increasing singularity $\mathcal{F}$ on $X$ also implies that every strictly decreasing sequence on $Y$ must converge. We now apply Lemmas 5.4 and 5.5. If $\xi \neq \eta$, then $\mathcal{M}=\mathcal{F} \times \dot{y}$, and if $\xi=\eta>\omega$, then $f(\mathcal{M})$ and $f(\mathcal{F} \times \dot{y})$ have the same limit in $[0,1]$ for all $f \in C I^{*}(X \times Y)$. Thus, under all circumstances, $\left(\sigma^{\prime}\right)^{-1}(\alpha)$ is a singleton, and the proof of the theorem is complete.

For totally ordered spaces $X$ and $Y$, one can show that if $X \times Y$ is pseudo-compact, then neither $X$ nor $Y$ has a singularity of order $\omega$. Thus it follows by Theorem 5.6 that if $X \times Y$ is pseudo-compact, $\beta_{0} X \times \beta_{o} Y=\beta_{o}(X \times Y)$. The converse is false, as we showed at the end of Section 4.

\section{REFERENCES}

[1] BLATTER, J., Order compactifications of totally ordered topological spaces, J. Approximation Theory 13 (1975), 56-65.

[2] CHOE, T. and PARK, Y., Wallman's type order compactification, Pacific J. Math. 82 (1979), 339-347.

[3] FLETCHER, P. and LINDGREN, W., Quasi-uniform spaces, Lect. Notes in Pure and Appl. Math., Vol. 77, Marcel Dekker, Inc., New York, 1982.

[4] GAMON, M. and KENT, D., A note on ordered planes, Internat. J. Math. \& Math. Sci. 15 (1992), 199202.

[5] GLICKSBERG, I., Stone-Čech compactification of products, Trans. Amer. Math. Soc. 90 (1959), 369382.

[6] KENT, D., On the Wallman ordered compactification, Pacific J. Math. 118 (1985), 159-163.

[7] KENT, D. and RICHMOND, T., Ordered compactifications of totally ordered spaces, Internat. J. Math. \& Math. Sci. 11 (1988), 683-694.

[8] NACHBIN, J., Topology and Order, Van Nostrand Math. Studies, No. 4, Princeton, NJ (1965). 


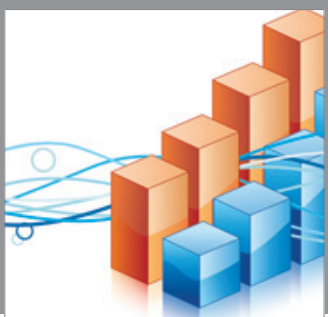

Advances in

Operations Research

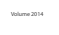

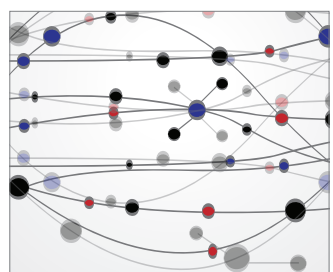

\section{The Scientific} World Journal
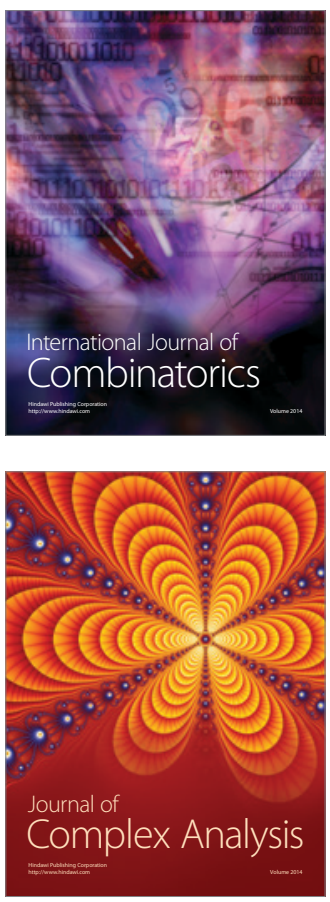

International Journal of

Mathematics and

Mathematical

Sciences
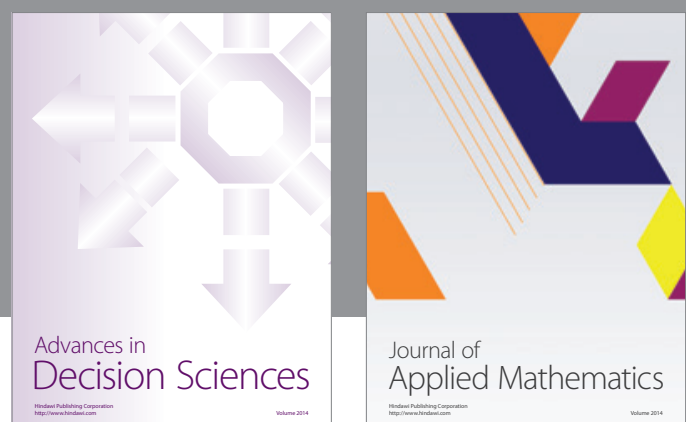

Journal of

Applied Mathematics
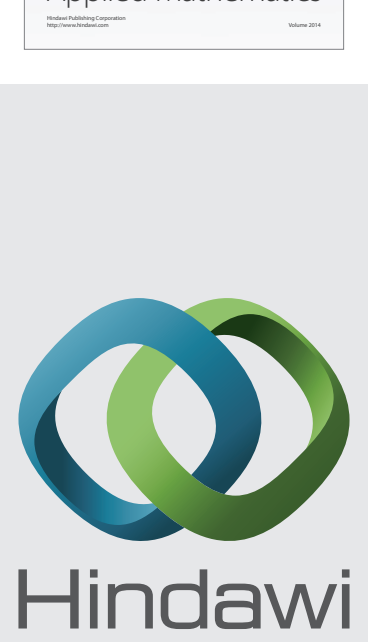

Submit your manuscripts at http://www.hindawi.com
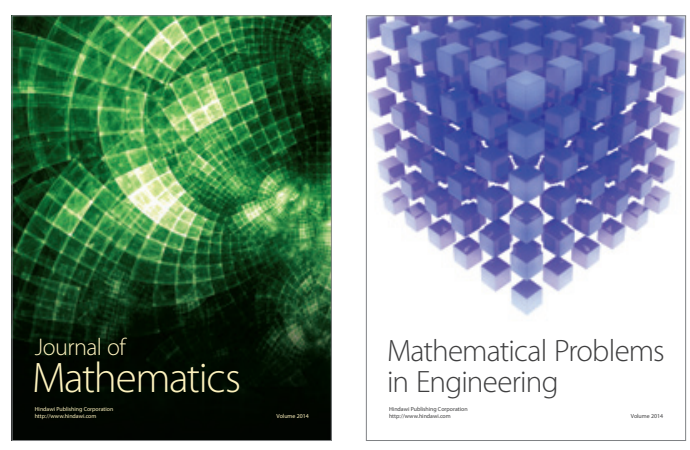

Mathematical Problems in Engineering
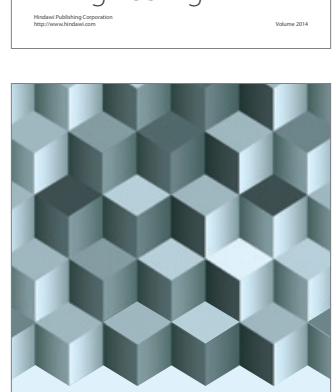

Journal of

Function Spaces
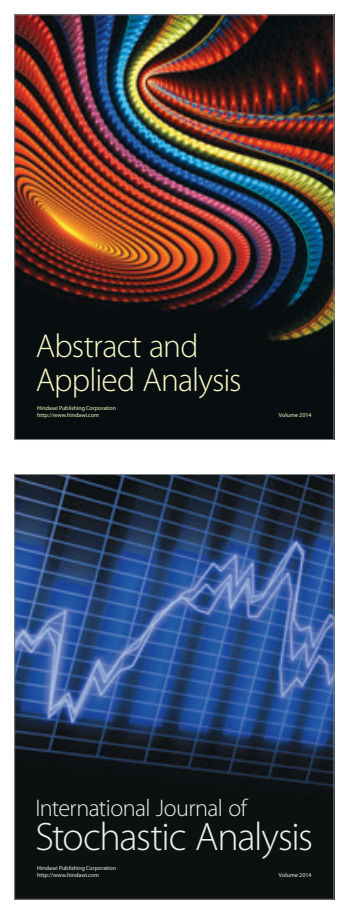

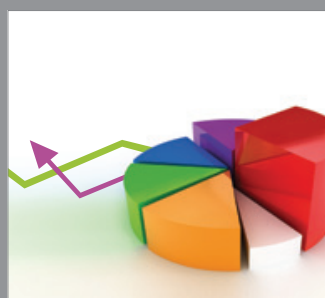

ournal of

Probability and Statistics

Promensencen
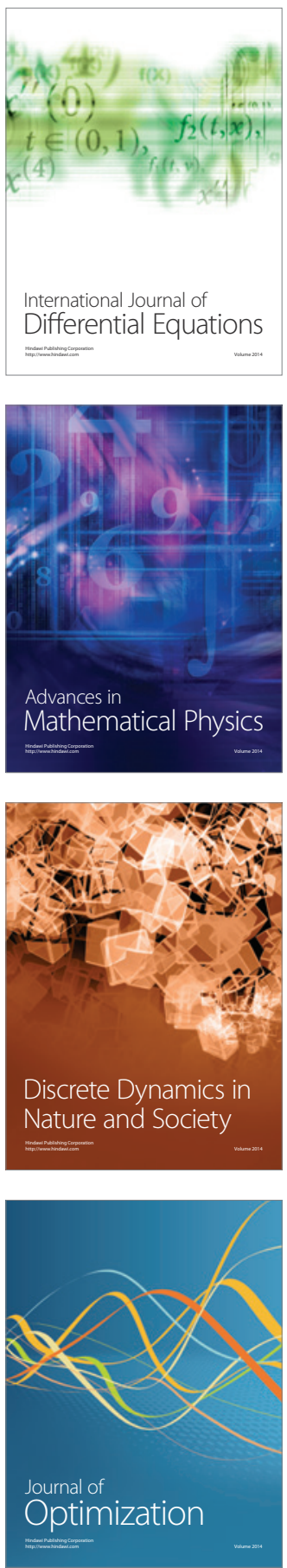